\title{
Computation of Some Geometric Properties for New Nonlinear PDE Models
}

\author{
Nassar Hassan Abdel-All ${ }^{1}$, Mohammed Abdullah Abdullah Hamad ${ }^{1}$, \\ Mohammed Abdullah Abdel-Razek ${ }^{1}$, Amal Aboelwafa Khalil ${ }^{2}$ \\ ${ }^{1}$ Department of Mathematics, Faculty of Science, Assiut University, Assiut, Egypt \\ ${ }^{2}$ Department of Mathematics, Faculty of Science, Sohag University, Sohag, Egypt \\ E-mail: amalaboelwafay@yahoo.com \\ Received March 16, 2011; revised March 25, 2011; accepted March 28, 2011
}

\begin{abstract}
The purpose of the present work is to construct new geometrical models for motion of plane curve by Darboux transformations. We get nonlinear partial differential equations (PDE). We have obtained the exact solutions of the resulting equations using symmetry groups method. Also, the Gaussian and mean curvatures of Monge form of the soliton surfaces have been calculated and discussed.
\end{abstract}

Keywords: Motion of Curve, Darboux Transformations, Gaussian and Mean Curvatures, Symmetry Groups

\section{Introduction}

Kinematics of moving curves in two dimension is formulated in terms of intrinsic geometries. The velocity is assumed to be local in the sense that it is a functional of the curvature and its derivatives. Plane curves have received a great deal of attention from mathematics, physics, biology, dynamic system, image processing and computer vision [1,2]. Evolution of plane curve can be understand as a deformation of a plane curve in arbitrary direction according to arbitrary a mount in a continuously process that has the time as a parameter. Physically this arbitrary a mount is a function of velocity, so this process create a sequence of evolving planer curve moving by a funcion of velocity, the type of the motion (evolution) of this family of planer curves classified depend on our choice of velocity function.

Let $r(s, t)$ is the position vector of a curve $C$ moving in space and let $\{T, n, b\}$ denote respectively the unit tangent, principal normal and binormal vectors vary along $C$. If we introduce the darboux vector (see [3]),

$$
\Omega=\tau T+k b
$$

then the Serret-Frenet equations may be written as the following [4]:

$$
\begin{aligned}
& T_{s}=\Omega \times T=k n, \\
& n_{s}=\Omega \times n=\tau b-k T, \\
& b_{s}=\Omega \times b=-\tau n
\end{aligned}
$$

where $s$ is the arc length parameter along $C, k$ the curvature and $\tau$ the torsion. In the present moving curve context, the time $t$ enters into the system (2) as a sparameter. The general temporal evolution in which the triad $\{T, n, b\}$ remains orthonormal adopts the form (darboux formula) [3]

$$
\begin{aligned}
& T_{t}=\alpha n+\beta b, \\
& n_{t}=-\alpha T+\gamma b \\
& b_{t}=-\beta T-\gamma n
\end{aligned}
$$

where $\alpha$ is the geodesic curvature, $\beta$ is the normal curvature and $\gamma$ is the geodesic torsion. Here, it is required that the arc length and time derivatives commute. This implies inextensibility of $C$. Accordingly, the compatibility conditions $T_{s t}=T_{t s}, n_{s t}=n_{t s}$ and $b_{s t}=b_{t s}$, applied to the systems (2) and (3) yield

$$
\begin{aligned}
& \alpha_{s}=k_{t}+\beta \tau, \\
& \beta_{s}=k \gamma-\tau \alpha, \\
& \gamma_{s}=\tau_{t}-k \beta .
\end{aligned}
$$

If the velocity vector $v=r_{t}$ of a moving curve $C$ has the form

$$
v=\lambda T+\mu n+v b,
$$

then imposition of the condition $r_{t s}=r_{s t}$ yields

$$
\lambda_{s} t+\lambda t_{s}+\mu_{s} n+\mu n_{s}+v_{s} b+v b_{s}=\alpha n+\beta b .
$$

Substitute about Serret-Frenet equations 


$$
\begin{aligned}
& \lambda_{s}-\mu k=0, \\
& \lambda k+\mu_{s}-v \tau=\alpha, \\
& \mu \tau+v_{s}=\beta .
\end{aligned}
$$

The temporal evolution of the curvature $k$ and the torsion $\tau$ of the curve $C$ may now be expressed in terms of the components of velocity $\lambda, \mu$ and $v$ by substitution of (7) into (4) to obtain

$$
\begin{aligned}
& k_{t}=\left(\lambda k+\mu_{s}-v \tau\right)_{s}-\left(\mu \tau+v_{s}\right) \tau, \\
& \tau_{t}=\gamma_{s}+\left(\mu \tau+v_{s}\right) k,
\end{aligned}
$$

where

$$
\gamma=\frac{1}{k}\left[\left(\mu \tau+v_{s}\right)_{s}+\tau\left(\lambda k+\mu_{s}-v \tau\right)\right] .
$$

Motion in a plane occurs if $v=0$ and $\tau=0$. Then Equation (8) becomes

$$
k_{t}=\left(\lambda k+\mu_{s}\right)_{s}=\lambda_{s} k+\lambda k_{s}+\mu_{s s} .
$$

From Equation (7), we have $\lambda=\int \mu k \mathrm{~d} s$ then Equation (10) becomes

$$
k_{t}=\mu_{s s}+\mu k^{2}+k_{s} \int \mu k \mathrm{~d} s .
$$

If we take $\int \mu k \mathrm{~d} s=\int \mathrm{d} F(k)=F(k)$ then $\mu k \mathrm{~d} s=\mathrm{d} F(k) \Rightarrow \mu=\frac{\mathrm{d} F(k)}{k \mathrm{~d} s}=\frac{k_{s}}{k} F(k)^{\prime}$, hence the equation (11) becomes

$$
k_{t}=\left(\frac{k_{s}}{k} F(k)^{\prime}\right)_{s s}+k^{2}\left(\frac{k_{s}}{k} F(k)^{\prime}\right)+k_{s} F(k) .
$$

\section{Symmetry Group}

Now, we want to present the most general Lie group of point transformations, which apply on obtaining equations

Definition 1. We consider a scalar $m$-th order $P D E$ represented by

$$
\Delta\left(s, k^{(m)}\right)=0, \text { where } m \text { is natural number }
$$

where $s=\left(s_{i}\right), i=1, \cdots, p$ is a vector for which the components $s_{i}$ are independent variables and $k=\left(k_{j}\right)$, $j=1, \cdots, q$ is a vector cosest of $k_{j}$ dependent variables, and $k^{(m)}=\frac{\partial^{m} k}{\partial s^{m}}$. The infinitesimal generator of the one-parameter Lie group of transformations for equation (13) is

$$
I X=\sum_{i=1}^{p} \zeta^{i}(s, k) \frac{\partial}{\partial s^{i}}+\sum_{\Omega=1}^{q} \phi^{\varkappa}(s, k) \frac{\partial}{\partial k^{\varkappa}},
$$

where $\zeta^{i}(s, k), \phi^{\varkappa}(s, k)$ are the infinitesimals, and the $m_{t h}$ prolongation of the infinitesimal generator (14) is (see [5-8])

$$
\operatorname{lp} r^{(m)} X=X+\sum_{\Omega=1}^{q} \sum_{j} \phi_{j}^{\varkappa}\left(s, k^{(m)}\right) \frac{\partial}{\partial k_{j}^{\varkappa}},
$$

where

$$
l \phi_{j}^{\varkappa}\left(s, k^{(m)}\right)=D_{j}\left(\phi^{\varkappa}-\sum_{i=1}^{p} \zeta^{i} k_{i}^{\varkappa}\right)+\sum_{i=1}^{p} \zeta^{i} k_{j, i}^{\varkappa} .
$$

and $D$ is the total derivative operator defined by

$$
D_{j}=\frac{\partial}{\partial s^{j}}+k_{j}^{\varkappa} \frac{\partial}{\partial k^{\varkappa}}+k_{i j}^{\varkappa} \frac{\partial}{\partial k_{i}^{\varkappa}}+\cdots, k_{j}=\frac{\partial k}{\partial s^{j}}, j=1, \cdots, p
$$

A vector field $\boldsymbol{X}$ is an infinitesimal symmetry of the system of differential Equations (13) if and only if it satisfies the infinitesimal invariance condition

$$
\left.\operatorname{lp} r^{(m)} \boldsymbol{X}(\Delta)\right|_{\Delta=0}=0
$$

\section{Soliton Geometry}

In this paper, we construct the soliton surfaces associated with the single soliton solutions (similarity solution) of the Equation (12). For this purpose, if $k=k(s, t)$ is a similarity solution of Equation (2), we have a solution surface $\sigma$ given from the Monge patch $f=(s, t, k(s, t))$. The tangent vectors $f_{s}, f_{t}$ for the soliton surface $\sigma$ are given by

$$
\begin{aligned}
& f_{s}=\left(1,0, k_{s}\right), \\
& f_{t}=\left(0,1, k_{t}\right) .
\end{aligned}
$$

The normal unit vector field on the tangents $T_{p} \sigma$ is given by

$$
N=\frac{f_{s} \wedge f_{t}}{\left|f_{s} \wedge f_{t}\right|}
$$

The 1st and 2nd fundamental forms on $\sigma$ are defined respectively by

$$
\begin{gathered}
I=\langle\mathrm{d} f, \mathrm{~d} f\rangle=g_{11} \mathrm{~d} s^{2}+2 g_{12} \mathrm{~d} s \mathrm{~d} t+g_{22} \mathrm{~d} t^{2}, \\
I I=\langle-\mathrm{d} f, \mathrm{~d} N\rangle=L_{11} \mathrm{~d} s^{2}+2 L_{12} \mathrm{~d} s \mathrm{~d} t+L_{22} \mathrm{~d} t^{2},
\end{gathered}
$$

where the tensor $g_{i j}$ and $L_{i j}$ are given by

$$
\begin{aligned}
& g_{11}=\left\langle f_{s}, f_{s}\right\rangle, g_{12}=\left\langle f_{s}, f_{t}\right\rangle, g_{22}=\left\langle f_{t}, f_{t}\right\rangle, \\
& L_{11}=\left\langle f_{s s}, N\right\rangle, L_{12}=\left\langle f_{s t}, N\right\rangle, L_{22}=\left\langle f_{t t}, N\right\rangle .
\end{aligned}
$$

The Gauss equations associated with $\sigma$ are

$$
\begin{aligned}
& f_{s s}=\Gamma_{11}^{1} f_{s}+\Gamma_{11}^{2} f_{t}+L_{11} N, \\
& f_{s t}=\Gamma_{12}^{1} f_{s}+\Gamma_{12}^{2} f_{t}+L_{12} N, \\
& f_{t t}=\Gamma_{22}^{1} f_{s}+\Gamma_{22}^{2} f_{t}+L_{22} N,
\end{aligned}
$$


while the Weingarten equations comprise

$$
\begin{aligned}
& N_{s}=\frac{g_{12} L_{12}-g_{22} L_{11}}{g} f_{s}+\frac{g_{12} L_{11}-g_{11} L_{12}}{g} f_{t}, \\
& N_{t}=\frac{g_{12} L_{22}-g_{22} L_{12}}{g} f_{s}+\frac{g_{12} L_{12}-g_{11} L_{22}}{g} f_{t},
\end{aligned}
$$

where

$$
g=\left|f_{s} \wedge f_{t}\right|^{2}=g_{11} g_{22}-g_{12}^{2} .
$$

The functions $\Gamma_{j \lambda}^{i}$ in (23) are the usual Christoffel symbols given by the relations

$$
\Gamma_{j \lambda}^{i}=\frac{1}{2} g^{i l}\left(g_{j l, \lambda}+g_{\lambda l, j}-g_{j \lambda, l}\right)
$$

The compatibility conditions $\left(f_{s s}\right)_{t}=\left(f_{s t}\right)_{s}$ and $\left(f_{s t}\right)_{t}=\left(f_{t t}\right)_{s}$ applied to the linear Gauss system (23) produce the nonlinear Mainardi-Codazzi system

$$
\begin{aligned}
& \left(\frac{L_{11}}{\sqrt{g}}\right)_{t}-\left(\frac{L_{12}}{\sqrt{g}}\right)_{s}+\frac{L_{11}}{\sqrt{g}} \Gamma_{22}^{2}-2 \frac{L_{12}}{\sqrt{g}} \Gamma_{12}^{2}+\frac{L_{22}}{\sqrt{g}} \Gamma_{11}^{2}=0, \\
& \left(\frac{L_{22}}{\sqrt{g}}\right)_{s}-\left(\frac{L_{12}}{\sqrt{g}}\right)_{t}+\frac{L_{11}}{\sqrt{g}} \Gamma_{22}^{1}-2 \frac{L_{12}}{\sqrt{g}} \Gamma_{12}^{1}+\frac{L_{22}}{\sqrt{g}} \Gamma_{11}^{1}=0,
\end{aligned}
$$

or, equivalently,

$$
\begin{aligned}
& L_{11 t}-L_{12 s}=L_{11} \Gamma_{12}^{1}+L_{12}\left(\Gamma_{12}^{2}-\Gamma_{11}^{1}\right)-L_{22} \Gamma_{11}^{2}, \\
& L_{12 t}-L_{22 s}=L_{11} \Gamma_{22}^{1}+L_{12}\left(\Gamma_{22}^{2}-\Gamma_{12}^{1}\right)-L_{22} \Gamma_{12}^{2},
\end{aligned}
$$

The Gaussian and mean curvatures at the regular points on the soliton surface are given by respectively

$$
\begin{gathered}
K=k_{1} k_{2}=\frac{L}{g}=\frac{L_{11} L_{22}-L_{12}^{2}}{g_{11} g_{22}-g_{12}^{2}}, g \neq 0 \\
H=\frac{1}{2}\left(k_{1}+k_{2}\right)=\frac{1}{2} \frac{L_{11} g_{22}-2 L_{12} g_{12}+L_{22} g_{11}}{g_{11} g_{22}-g_{12}^{2}} .
\end{gathered}
$$

where $g=\operatorname{det}\left(g_{i j}\right), L=\operatorname{det}\left(L_{i j}\right)$ and $k_{1}, k_{2}$ are the principal curvatures. The surface for which $K=0$ is called parabalic surface, but if $k_{1}=0$ and $k_{2}=$ constant or $k_{1}=$ constant and $k_{2}=0$, we have surface semi round semi flat (cylinderial like surface). The integrability conditions for the systems (2) and (3) are equivalente to the Mainardi-Codazzi system of PDE (27). This give a geometric interpretation for the surface defined by the variables $s, t$ to be a soliton surface $[9,10]$.

\section{Applications}

\subsection{Case I: $F(k)=-k$}

The Equation (12) becomes

$$
\Delta_{1}=k^{3} k_{t}-3 k k_{s} k_{s s}+k^{2} k_{s s s}+2 k_{s}^{3}+2 k^{4} k_{s}=0 .
$$

The infinitesimal point symmetry of Equations (31) will be a vector field of the general form

$$
X=\zeta \frac{\partial}{\partial s}+\eta \frac{\partial}{\partial t}+\phi \frac{\partial}{\partial k}
$$

on $M=R^{3}$; our task is to determine which particular coefficient functions $\zeta, \eta$ and $\phi$ are functions of the variables $s, t$ and $k$ and will produce infinitesimal symmetries. In order to apply condition (18), we must compute the third order prolongation of $X$, which is the vector field

$$
\operatorname{pr}^{(3)} X=\zeta \frac{\partial}{\partial s}+\eta \frac{\partial}{\partial t}+\phi \frac{\partial}{\partial k}+\sum_{J} \phi^{J} \frac{\partial}{\partial k_{J}},
$$

whose coefficients, in view of (31), are given by the explicit formulae

$$
\begin{aligned}
& \phi^{s}=D_{s}\left(\phi-\zeta k_{s}-\eta k_{t}\right)+\zeta k_{s s}+\eta k_{s t}, \\
& \phi^{t}=D_{t}\left(\phi-\zeta k_{s}-\eta k_{t}\right)+\zeta k_{s t}+\eta k_{t t}, \\
& \phi^{s s}=D_{s s}\left(\phi-\zeta k_{s}-\eta k_{t}\right)+\zeta k_{s s s}+\eta k_{s s t}, \\
& \phi^{s s s}=D_{s s s}\left(\phi-\zeta k_{s}-\eta k_{t}\right)+\zeta k_{s s s}+\eta k_{s s s t} .
\end{aligned}
$$

The vector field $X$ is an infinitesimal symmetry of the Equation (31) if and only if

$$
\begin{aligned}
\operatorname{pr}^{(3)} X\left(\Delta_{1}=0\right)= & k^{3} \phi^{t}+3 k^{2} \phi-3 k_{s} k_{s s} \phi-3 k k_{s s} \phi^{s} \\
& -3 k k_{s} \phi^{s s}+2 k k_{s s s} \phi+k^{2} \phi^{s s s} \\
& +6 k_{s}^{2} \phi^{s}+8 \phi k^{3} k_{s}+2 k^{4} \phi^{s}=0 .
\end{aligned}
$$

Substituting the prolongation Formulae (34), and equa- ting the coefficients of the independent derivative mono- mials to zero, leads to the infinitesimal determining equations which together with their differential conse- quences reduce to the system

$$
\phi=-\frac{1}{2} k \eta_{t}, \zeta_{s}=\frac{1}{2} \eta_{t}, \zeta_{k}=\eta_{k}=\eta_{s}=\zeta_{t}=0 .
$$

The general solution of this system is readily found

$$
\zeta=\frac{1}{2} c_{3} s+c_{1}, \eta=c_{3} t+c_{2}, \phi=-\frac{1}{2} c_{3} k,
$$

where the coefficients $c_{i}$ are arbitrary constants. Therefore, Equation (31) admits the three-dimensional Lie algebra of infinitesimal symmetries, spanned by the three vector fields

$$
X_{1}=\frac{\partial}{\partial s}, X_{2}=\frac{\partial}{\partial t}, X_{3}=\frac{1}{2} s \frac{\partial}{\partial s}+t \frac{\partial}{\partial t}-\frac{1}{2} k \frac{\partial}{\partial k} \text {. }
$$

The combination of space and time translations $\left(X_{1}+X_{2}\right)$ lead to a reduction of (31) to an ordinary differential equation (ODE) by the transformation $y=s-c t$ and $k=w(y)$ where $c$ is the speed of the travelling wave. That is 


$$
w^{2} w^{\prime \prime \prime}-3 w w^{\prime} w^{\prime \prime}+-c w^{3} w^{\prime}+2 w^{\prime 3}+2 w^{4} w^{\prime}=0 .
$$

Now, the solution of the Equation (39) is,

$$
\int \pm \frac{1}{w \sqrt{-2 \ln (w) c_{2}-w^{2}+2 c w+2 c_{1}}} \mathrm{~d} w-y+c_{3}=0,
$$

where $c_{1}, c_{2}$ and $c_{3}$ are the integration constants, if we consider it equal zero, hence the solution of Equation (39) becomes

$$
w= \pm \frac{2 c}{c^{2} y^{2}+1}= \pm \frac{2 c}{c^{2}(s-c t)^{2}+1} .
$$

this solution is a similar solution to Equation (31), This solution is in the Monge form $w=w(y)=w(s, t)$ which define a regular surface as show in Figure 1 $(c=1,1 \leq s \leq 5,0.1 \leq t \leq 2)$.

This surface is a soliton surface $(1+1)$. From (29) and (30), one can see that the Gaussian and mean curvatures of the soliton surface ( ) are given by respectively

$$
K=0, \quad H=\frac{4 \Gamma_{1}^{3}\left(-1+3 t^{2}-6 t s+3 s^{2}\right)}{\sqrt{\Gamma_{1}^{4}+32(t-s)^{2} \Gamma_{2}}},
$$

where

$$
\begin{aligned}
\Gamma_{1}= & 1+t^{2}-2 t s+s^{2} \\
\Gamma_{2}= & 1+t^{8}-8 t^{7} s+36 s^{2}+6 s^{4}+4 s^{6}+s^{8}+4 t^{6}\left(1+7 s^{2}\right) \\
& -8 t^{5} s\left(3+7 s^{2}\right)-8 t^{3} s\left(3+10 s^{2}+7 s^{4}\right) \\
& +t^{4}\left(6+60 s^{2}+70 s^{4}\right)-8 t s\left(9+3 s^{2}+3 s^{4}+s^{6}\right) \\
& +4 t^{2}\left(9+9 s^{2}+15 s^{4}+7 s^{6}\right) .
\end{aligned}
$$

The symmetry generator $X_{3}=\frac{1}{2} s \frac{\partial}{\partial s}+t \frac{\partial}{\partial t}-\frac{1}{2} k \frac{\partial}{\partial k}$ leads to invariants $y=\frac{t}{s^{2}}$ and $w=s k$. These the invariants transform Equation (31) to the following ODE,

$$
\begin{aligned}
& 8 y^{3} w^{2} w^{\prime \prime \prime}+\left(36 y^{2} w^{2}-24 y^{3} w w^{\prime}\right) w^{\prime \prime} \\
& +\left(24 y w^{2}-w^{3}-36 y^{2} w w^{\prime}+16 y^{3} w^{2}+4 y w^{4}\right) w^{\prime} \\
& +2\left(w^{2}+1\right) w^{3}=0
\end{aligned}
$$

The numerical solution of Equation (44) is shown in Figure 2 (intial condition $w(1)=1, w^{\prime}(1)=2$ and $\left.w^{\prime \prime}(1)=3\right)$.
4.2. Case II: $F(k)=\frac{1}{k}$

In this case Equation (12), becomes

$$
k^{5} k_{t}+k^{2} k_{s s s}-9 k k_{s} k_{s s}+12 k_{s}^{3}=0,
$$

Lie point symmetry for this equation is given by

$$
\begin{array}{ll}
X_{1}=\frac{\partial}{\partial s}, & X_{2}=\frac{\partial}{\partial t}, \\
X_{3}=s \frac{\partial}{\partial s}-k \frac{\partial}{\partial k}, & X_{4}=t \frac{\partial}{\partial t}+\frac{1}{3} k \frac{\partial}{\partial k},
\end{array}
$$

The combination $X=X_{1}+c X_{2}=\frac{\partial}{\partial s}+c \frac{\partial}{\partial t}$ gives rise to travelling wave solutions a wave speed $c$. The vector field $X$ has invariants $y=s-c t$ and $w=k$ which reduces (45) to the ODE

$$
w^{2} w^{\prime \prime \prime}-9 w w^{\prime} w^{\prime \prime}-c w^{5} w^{\prime}+12 w^{\prime 3}=0 .
$$

Now, solving the equation with the Lie symmetry spanned by

$$
\begin{aligned}
& Y_{1}=\frac{\partial}{\partial y}, \\
& Y_{2}=y \frac{\partial}{\partial y}-\frac{2}{3} w \frac{\partial}{\partial w} .
\end{aligned}
$$

If we take the vector field $Y_{2}$ we obtain solution $w=\rho y^{-\frac{2}{3}}$, and substituting $w$ in Equation (47) we get $\rho=\left(-\frac{2}{9 c}\right)^{\frac{1}{3}}$ and the solution

$$
k=\frac{\left(-\frac{2}{9 c}\right)^{\frac{1}{3}}}{(s-c t)^{\frac{2}{3}}}
$$

Remark 1. For regularity the parameters of the soliton surface must be satisfied $s \neq c t$, i.e., for $s=c t$ we have singularity (cuspidedge) as shown in Figure 3.

The Gaussian and mean curvatures respectively are (shown in Equation (50))

If we take the vector field $X_{1}+X_{3}$ we here the inriants $y=t$ and $k=\frac{1}{1+s} w$, that is $w^{\prime}=0$ then $w=$ constant and

$$
\begin{aligned}
& K=0 \\
& H=\frac{270 \sqrt[3]{6}(s+t)^{\frac{7}{3}}}{\sqrt{81(s+t)^{\frac{10}{3}}+8(6)^{\frac{2}{3}}}\left(8(6)^{\frac{2}{3}}+81 t^{3}(t+s)^{\frac{1}{3}}+243 t^{2} s(t+s)^{\frac{1}{3}}+243 t s^{2}(s+t)^{\frac{1}{3}}+81 s^{3}(s+t)^{\frac{1}{3}}\right.} .
\end{aligned}
$$



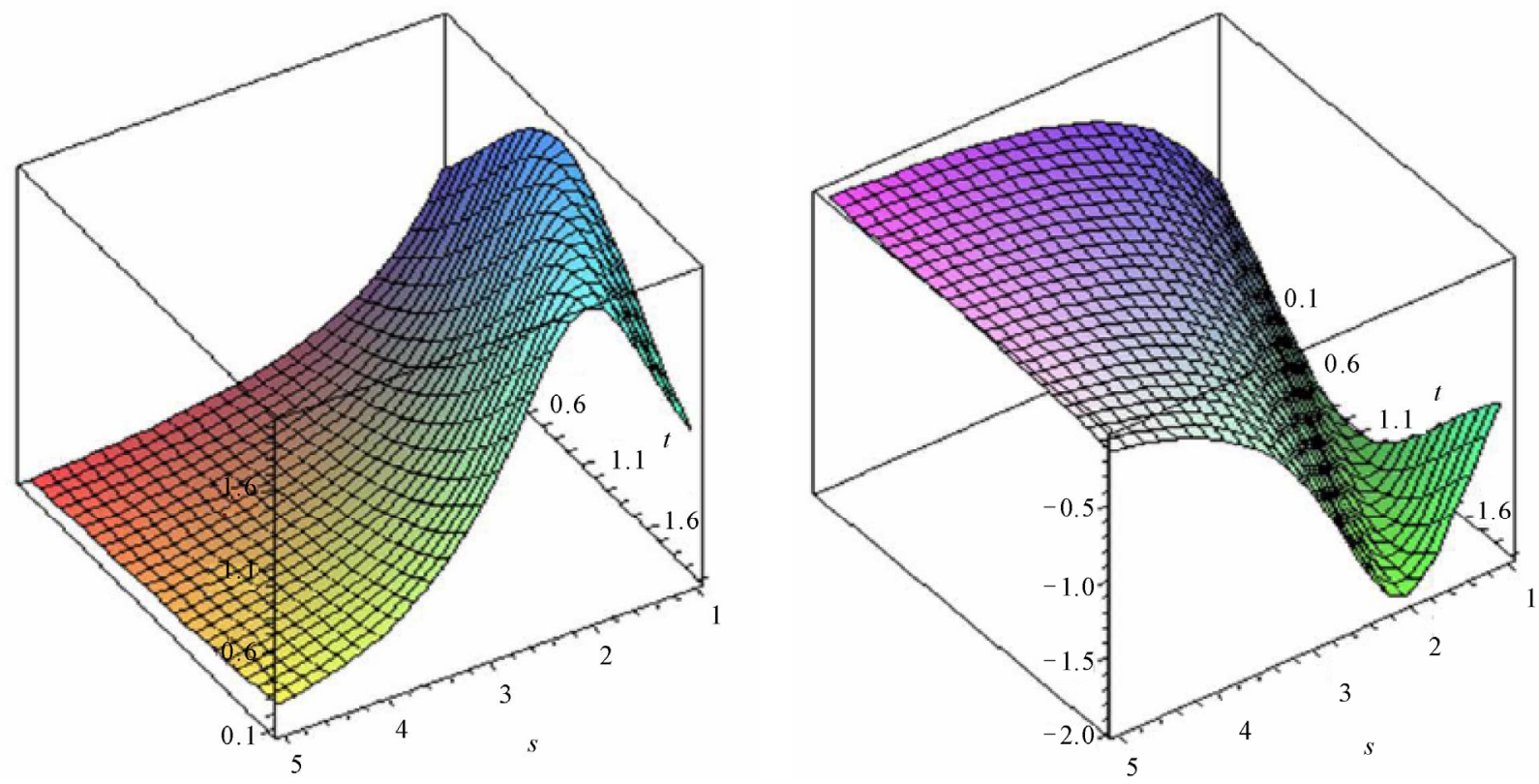

Figure 1. Soliton surfaces of (41).

$$
k=\frac{a}{1+s},
$$

thus we have Figure 4.

The vector field $X_{4}$ leads to the invariants $y=s$ and the tarnsformation $k=t^{\frac{1}{3}} w$ reduces (45) to an ODE in the form

$$
w^{6}-27 w w^{\prime} w^{\prime \prime}+3 w^{2} w^{\prime \prime \prime}+36 w^{\prime 3}=0,
$$

this equation can be solved numericaly (intial condition $w(0)=2, w^{\prime}(0)=2$ and $\left.w^{\prime \prime}(0)=3\right)$ as shown in Figure 5 .

\subsection{Case III: $F(k)=\frac{1}{k^{2}}$}

In this case Equation (12) takes the form

$$
k^{6} k_{t}+2 k^{2} k_{s s s}-24 k k_{s} k_{s s}+40 k_{s}^{3}+k^{4} k_{s}=0,
$$

Lie point symmetry of this equation is given by

$$
X_{1}=\frac{\partial}{\partial s}, X_{2}=\frac{\partial}{\partial t}, X_{3}=-s \frac{\partial}{\partial s}+t \frac{\partial}{\partial t}+k \frac{\partial}{\partial k},
$$

The combination $X=c X_{1}+X_{2}=c \frac{\partial}{\partial s}+\frac{\partial}{\partial t}$ gives rise to travelling wave solutions with wave speed $c$. The vector field $X$ has invariants $y=s-c t$ and $w=k$ which reduces (53) to

$$
-c w^{6} w^{\prime}+2 w^{2} w^{\prime \prime \prime}-24 w w^{\prime} w^{\prime \prime}+40 w^{\prime 3}+w^{4} w^{\prime}=0 .
$$

solving Equation (55) hence

$$
\int \frac{ \pm 6}{w^{2} \sqrt{-24 c_{2} w^{4}-18 c w^{2}+24 c_{1} w-9}} \mathrm{~d} w-y-c_{3}=0,
$$

If we take the integration constants to be zero hence the solution takes the form

$$
w= \pm \frac{2}{\sqrt{-8 c-y^{2}}}= \pm \frac{2}{\sqrt{-8 c-(s-c t)^{2}}},
$$

For regularity the parameters of the soliton surface must be satisfied $s-c t \neq \pm \sqrt{8 c_{1}}$ at $c_{1}=-c$, i.e., for $s-c t= \pm \sqrt{8 c_{1}}$ we have singularity (cuspidaledge) as shown in Figure 6.

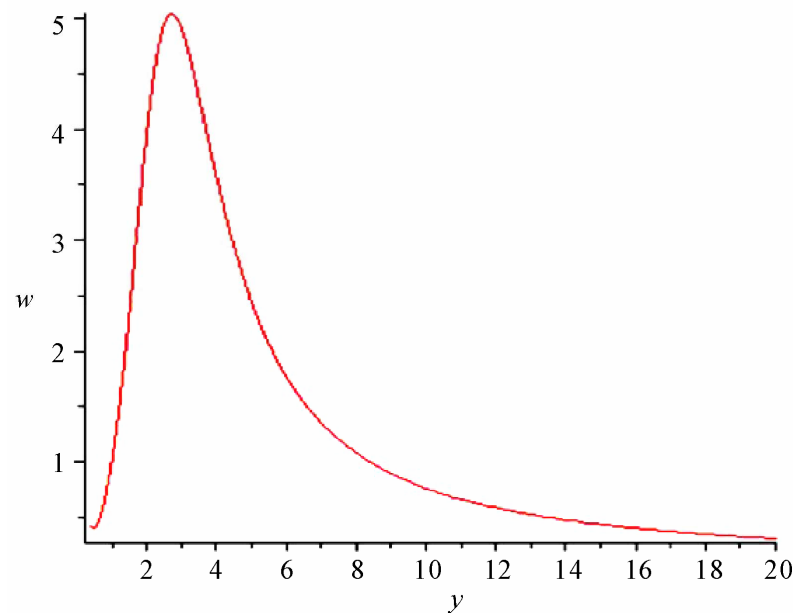

Figure 2. Numerical solution of (44). 


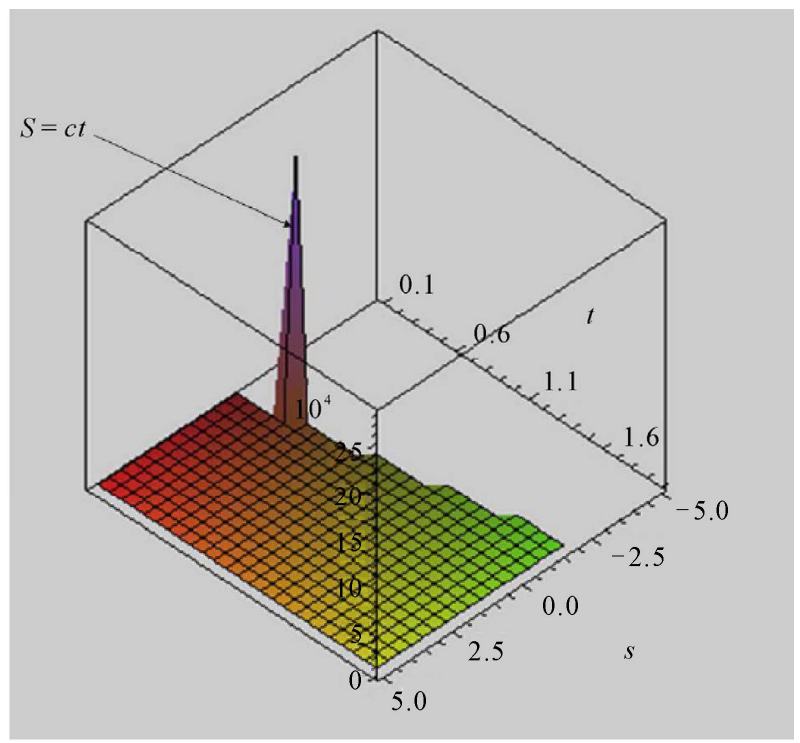

Figure 3. Soliton surfaces of (49).

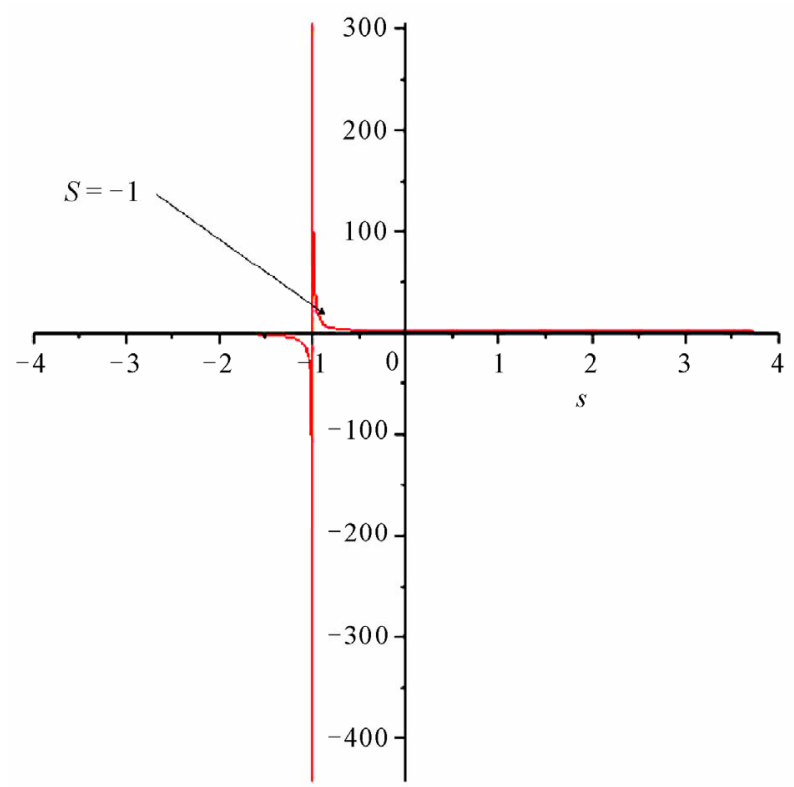

Figure 4. Solution of (51).

Gaussian and mean curvatures are

$$
K=0, H=\frac{4\left(-8+t^{2}+2 t s+s^{2}\right)\left(4+t^{2}+s^{2}+2 t s\right)}{\Gamma_{2} \sqrt{-\sqrt{\Gamma_{1}^{2}-8(s+t)^{2}}}}
$$

where

$$
\begin{aligned}
\Gamma_{1}= & -8+(s+t) \\
\Gamma_{2}= & t^{6}-8 t s+188 s^{2}-96 t s^{2}-24 s^{4}+6 t s^{5} \\
& +6 t^{4}(-4+t s)+t^{2}\left(188-96 t s+15 t^{2} s^{2}\right) \\
& +s^{2}\left(-4+s^{4}\right)+t^{2}\left(-4+15 s^{2}\right)
\end{aligned}
$$

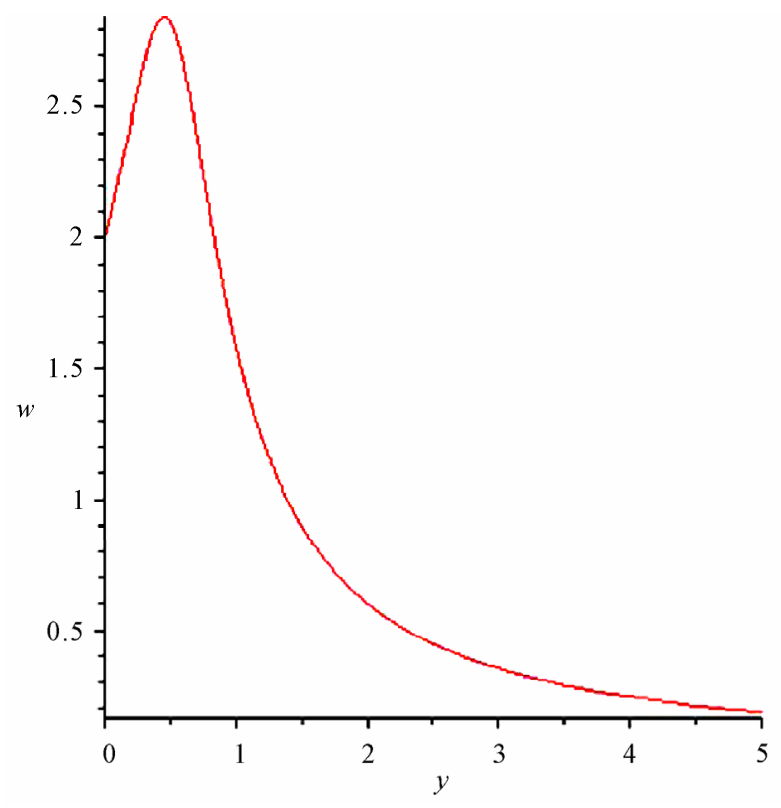

Figure 5. Numerical solution of (52).

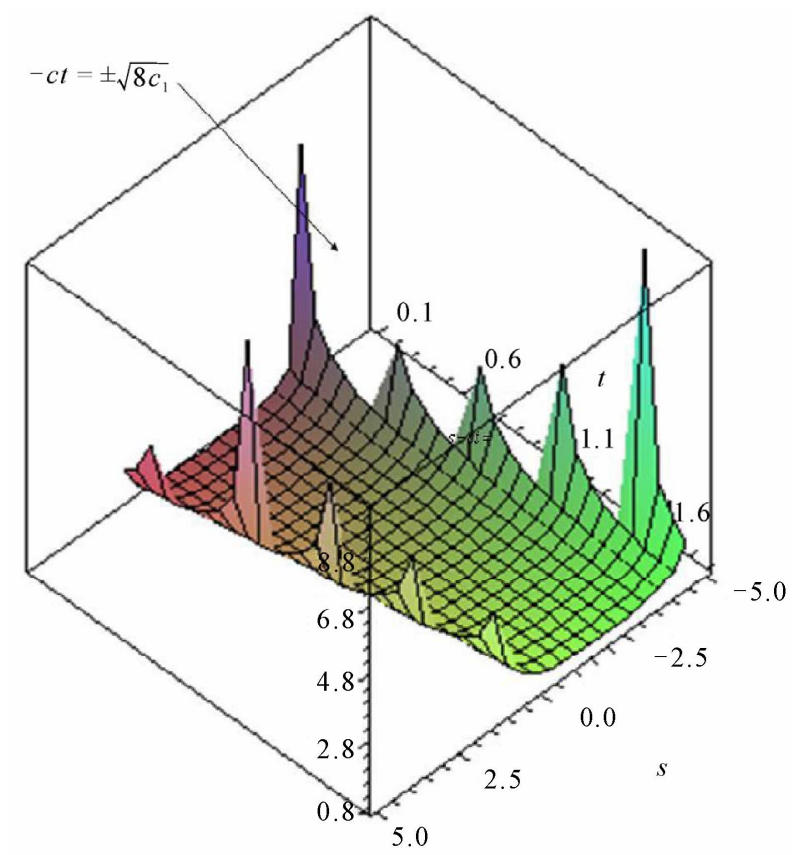

Figure 6. Soliton surface of (57).

The symmetry generator $X_{3}=-s \frac{\partial}{\partial s}+t \frac{\partial}{\partial t}+k \frac{\partial}{\partial k}$ leads to the invariants $y=s t$ and $w=s k$. After some detailed and tedious calculations, (53) becomes ODE

$$
\begin{aligned}
& 2 y^{3} w^{2} w^{\prime \prime \prime}+\left(18 y^{2} w^{2}-24 y^{3} w w^{\prime}\right) w^{\prime \prime} \\
& +\left(w^{6}-72 y^{2} w w^{\prime}+36 y w^{2}\right. \\
& \left.+40 y^{3} w^{\prime 2}+y w^{4}-1\right) w^{\prime}-4 w^{3}=0
\end{aligned}
$$


The numerical solution of Equation (60) is shown in Figure 7 (intial condition $w(1)=1, w^{\prime}(1)=2$ and $w^{\prime \prime}(1)=3$ ).

4.4. Case IV: $F(k)=\frac{1}{k+1}$

In this case Equation (12) becomes

$$
\begin{aligned}
& k^{7} k_{t}+4 k^{6} k_{t}+6 k^{5} k_{t}+4 k^{4} k_{t}+k^{3} k_{t}+k^{4} k_{s s s} \\
& +2 k^{3} k_{s s s}+8 k k_{s}^{3}+2 k_{s}^{3}+12 k^{2} k_{s}^{3}-k^{3} k_{s}-2 k^{4} k_{s} \\
& -9 k^{3} k_{s} k_{s s}+k^{2} k_{s s s}-12 k^{2} k_{s} k_{s s} \\
& -3 k k_{s} k_{s s}-k^{5} k_{s}=0,
\end{aligned}
$$

Lie point symmetry of this equation is given by

$$
X_{1}=\frac{\partial}{\partial s}, X_{2}=\frac{\partial}{\partial t}
$$

The combination $X=c X_{1}+X_{2}=c \frac{\partial}{\partial s}+\frac{\partial}{\partial t}$ gives rise to travelling wave solutions with wave speed $c$. The vector field $X$ has invariants $y=s-c t$ and $w=k$

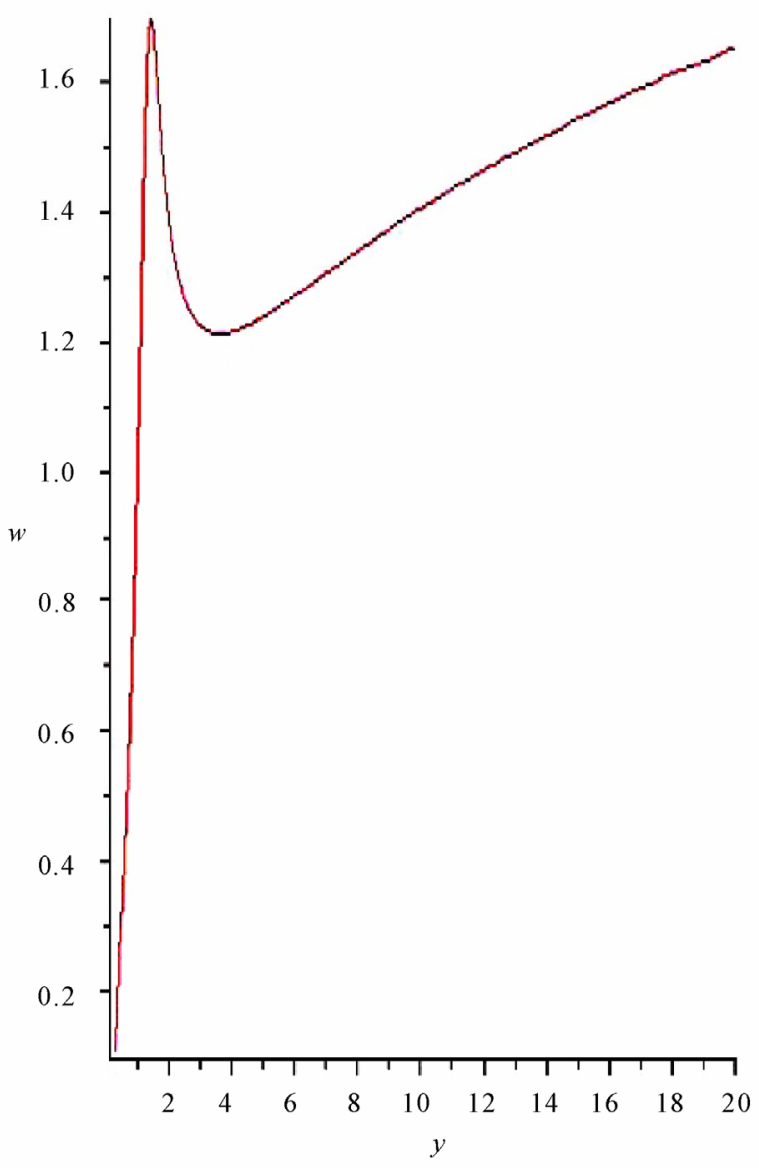

Figure 7. Numerical solution of (60). which reduces $(62)$ to

$$
\begin{aligned}
& -c w^{7} w^{\prime}-4 c w^{6} w^{\prime}-6 c w^{5} w^{\prime}-4 c w^{4} w^{\prime}-c w^{3} w^{\prime}+w^{4} w^{\prime \prime} \\
& +2 w^{3} w^{\prime \prime \prime}+8 w w^{\prime 3}+2 w^{\prime 3}+12 w^{2} w^{3}-w^{3} w^{\prime}-2 w^{4} w^{\prime} \\
& -9 w^{3} w^{\prime} w^{\prime \prime}+w^{2} w^{\prime \prime \prime}-12 w^{2} w^{\prime} w^{\prime \prime}-3 w w^{\prime} w^{\prime \prime}-w^{5} w^{\prime}=0
\end{aligned}
$$

solving the Equation (63) we get

$$
\int \frac{1}{w(w+1) \sqrt{\Psi}} \mathrm{d} w-y+c_{3}=0
$$

where

$$
\begin{aligned}
& \Psi=-3+4 w c_{1}-2 c_{2} w^{2} \ln (w)+2 c_{2} w^{2} \ln (w+1) \\
& +2 w^{2} \ln (w+1)+2 c_{1} w^{2}-2 w^{2} \ln (w)-2 c_{2} w \\
& -4 w \ln (w)-2 c w-2 w-4 c_{2} w \ln (w) \\
& +4 c_{2} w \ln (w+1)+4 w \ln (w+1)-2 \ln (w) \\
& -2 c_{2} \ln (w)-2 c-2 c_{2}+2 \ln (w+1) \\
& +2 c_{2} \ln (w+1)+2 c_{1},
\end{aligned}
$$

If we take $c_{1}=c, c_{2}=-1$ and $c_{3}=0$

$$
\int \frac{1}{w(w+1) \sqrt{-1+2 c w+2 c w^{2}}} \mathrm{~d} w-y=0,
$$

then

$$
\begin{aligned}
y= & \tan ^{-1}\left(\frac{1}{2} \frac{-2+4 w}{\sqrt{4 w^{2}+4 w-1}}\right) \\
& -\tan ^{-1}\left(\frac{1}{2} \frac{-6-4 w}{\sqrt{4(w+1)^{2}-4 w-5}}\right) .
\end{aligned}
$$

Hence, we have a soliton surface given by the implicit equation

$$
\begin{aligned}
s-c t= & \tan ^{-1}\left(\frac{1}{2} \frac{-2+4 w}{\sqrt{4 w^{2}+4 w-1}}\right) \\
& -\tan ^{-1}\left(\frac{1}{2} \frac{-6-4 w}{\sqrt{4(w+1)^{2}-4 w-5}}\right),
\end{aligned}
$$

Gaussian and mean curvatures of implicit surface are

$$
\begin{aligned}
& K=0, \\
& H=\frac{\left(1+c^{2}\right) w(1+w)\left(-1+4 w+18 w^{2}+12 w^{3}\right)}{2\left(1-\left(1+c^{2}\right)\left(w^{2}-2 w^{3}-11 w^{4}-12 w^{5}-4 w^{6}\right)\right)^{\frac{3}{2}}}
\end{aligned}
$$

This surface is illustrated as in Figure 8.

\subsection{Case V: $F(k)=\ln (k)$}

In this case Equation (12) becomes 


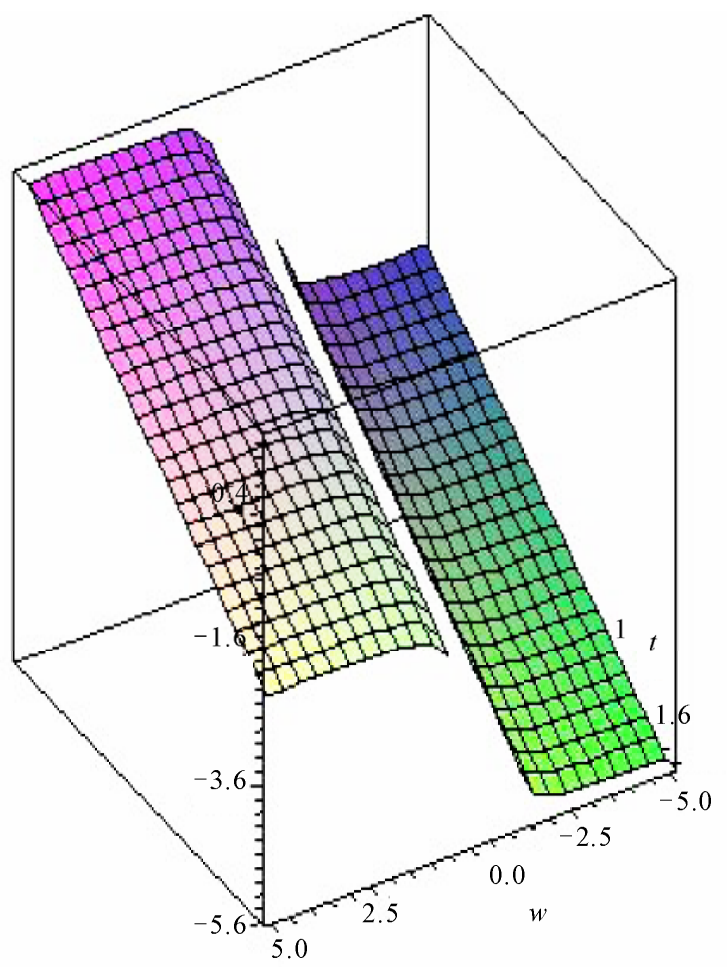

Figure 8. Soliton surface of (68).

$$
k^{4} k_{t}-k^{2} k_{s s s}+6 k k_{s} k_{s s}-6 k_{s}^{3}-k^{4} k_{s}-k^{4} k_{s} \ln (k)=0
$$

Lie point symmetry for this equation is given by

$$
X_{1}=\frac{\partial}{\partial s}, X_{2}=\frac{\partial}{\partial t}, X_{3}=(t+s) \frac{\partial}{\partial s}+t \frac{\partial}{\partial t}-k \frac{\partial}{\partial k},
$$

The combination $X=c X_{1}+X_{2}=c \frac{\partial}{\partial s}+\frac{\partial}{\partial t}$ gives rise to travelling wave solutions with wave speed $c$. The vector field $X$ has invariants $y=s-c t$ and $w=k$ wkich reduces $(70)$ to

$$
\begin{aligned}
& -w^{2} w^{\prime \prime \prime}+6 w w^{\prime} w^{\prime \prime} \\
& -\left(c w^{4}+6 w^{\prime 2}+w^{4}+w^{4} \ln (w)\right) w^{\prime}=0 .
\end{aligned}
$$

We have solved the Equation (72) with intial condition $\left(w(0)=2, w^{\prime}(0)=2\right.$ and $\left.w^{\prime \prime}(0)=3\right)$ numerically, which represented in Figures 9(a), (b). The Figure 9(a) represents the numerical solution at the forword wave, while Figure 9(b) at the backword wave.

\subsection{Case VI: $F(k)=\mathrm{e}^{-k}$}

In this case Equation (12) becomes

$$
\begin{aligned}
& e^{k} k^{4} k_{t}-3 k^{2} k s k_{s s}+k^{2} k_{s}^{3}+2 k k_{s}^{3}+k^{2} k_{s s s} \\
& -3 k k_{s} k_{s s}+2 k_{s}^{3}+k^{4} k_{s}-k^{3} k_{s}=0,
\end{aligned}
$$

Lie point symmetry for this equation is given by

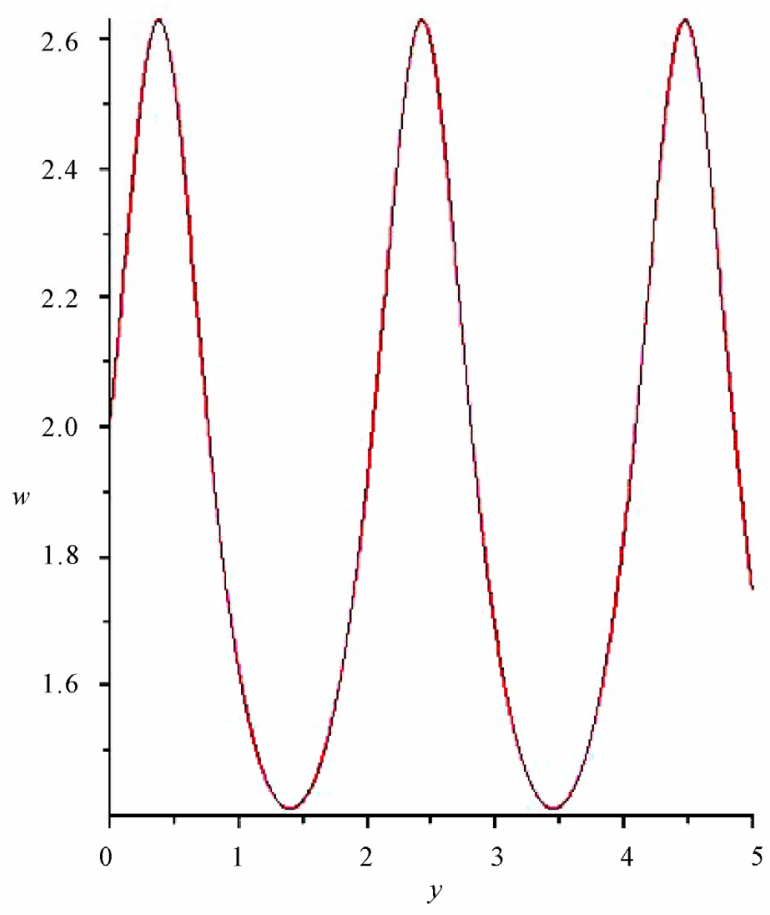

(a)

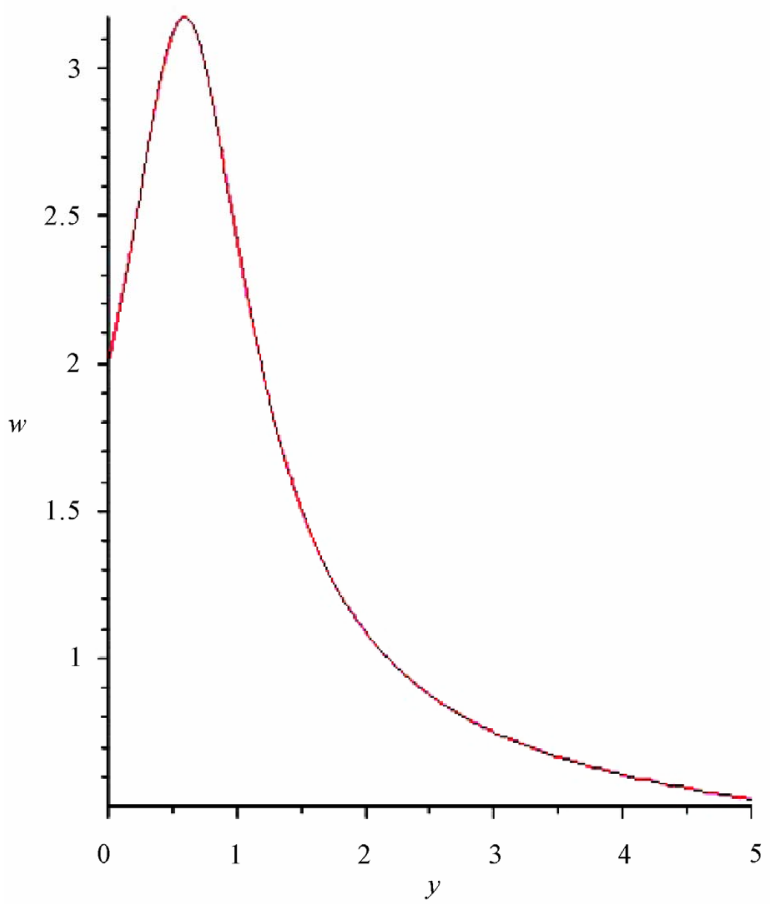

(b)

Figure 9. Numerical solution of (72).

$$
X_{1}=\frac{\partial}{\partial s}, X_{2}=\frac{\partial}{\partial t}
$$

the travelling wave solution is obtained by 
$X=c \frac{\partial}{\partial s}+\frac{\partial}{\partial t}$ by which (73) becomes the o.d.e (with the new independent variable $y=s-c t, c$ being the speed of the wave)

$$
\begin{aligned}
& w^{2} w^{\prime \prime \prime}-\left(3 w+3 w^{2}\right) w^{\prime} w^{\prime \prime} \\
& +\left(w^{2} w^{\prime 2}-c e^{w} w^{4}+2 w w^{\prime 2}++2 w^{\prime 2}+w^{4}-w^{3}\right) w^{\prime}=0,
\end{aligned}
$$

by numerical (intial condition $w(0)=2, w^{\prime}(0)=2$ and $w^{\prime \prime}(0)=3$, range of 0 to 5) Figures 10(a), (b), This solution represents a curve on the soliton surface $w=w(s-c t)$ and $y=s-c t$.

\subsection{Case VII: $F(k)=\sqrt{k}$}

In this case Equation (12) becomes

$$
8 k^{\frac{7}{2}} k_{t}-4 k^{2} k_{\text {sss }}+18 k k_{s} k_{s s}-15 k_{s}^{3}-12 k^{4} k_{s}=0,
$$

Lie point symmetry for this equation is given by

$$
X_{1}=\frac{\partial}{\partial s}, X_{2}=\frac{\partial}{\partial t}, X_{3}=\frac{2}{3} s \frac{\partial}{\partial s}+t \frac{\partial}{\partial t}-\frac{2}{3} k \frac{\partial}{\partial k},
$$

the travelling wave solution is obtained by $X=c \frac{\partial}{\partial s}+\frac{\partial}{\partial t}$ by which (76) becomes the ODE (with the new independent variable $y=s-c t, c$ being the speed of the wave)

$$
-4 w^{2} w^{\prime \prime \prime}+18 w w^{\prime} w^{\prime \prime}-\left(c w^{\frac{7}{2}}+15 w^{2}+12 w^{4}\right) w^{\prime}=0,
$$

solving the Equation (78) we get

$$
\int \pm \frac{\sqrt{w}}{2 \sqrt{-\sqrt{w}\left(c_{2} w^{\frac{7}{2}}+w^{\frac{9}{2}}+2 c w^{4}-c_{1} w^{3}\right)}} \mathrm{d} w-y-c_{3}=0,
$$

If we take the integration constants to be zero Equation (79) becomes

$$
y=-\frac{(\sqrt{w}+2 c)\left(-w^{2}+c w^{\frac{3}{2}}\right)}{3 c^{2} \sqrt{-\sqrt{w}\left(w^{\frac{9}{2}}+2 c w^{4}\right)}},
$$

we have a soliton surface given by the implicit equation

$$
s-c t=-\frac{(\sqrt{w}+2 c)\left(-w^{2}+c w^{\frac{3}{2}}\right)}{3 c^{2} \sqrt{-\sqrt{w}\left(w^{\frac{9}{2}}+2 c w^{4}\right)}},
$$

Gaussian and mean curvatures of implicit surface are

$$
\begin{aligned}
& K=0, \\
& H=\frac{-\left(1+c^{2}\right)(7 c+4 \sqrt{w})^{\frac{5}{2}}}{\left(1-4\left(1+c^{2}\right)\left(2 w^{\frac{7}{2}}+2 c w^{\frac{7}{2}}+w^{4}\right)\right)^{\frac{3}{2}}},
\end{aligned}
$$

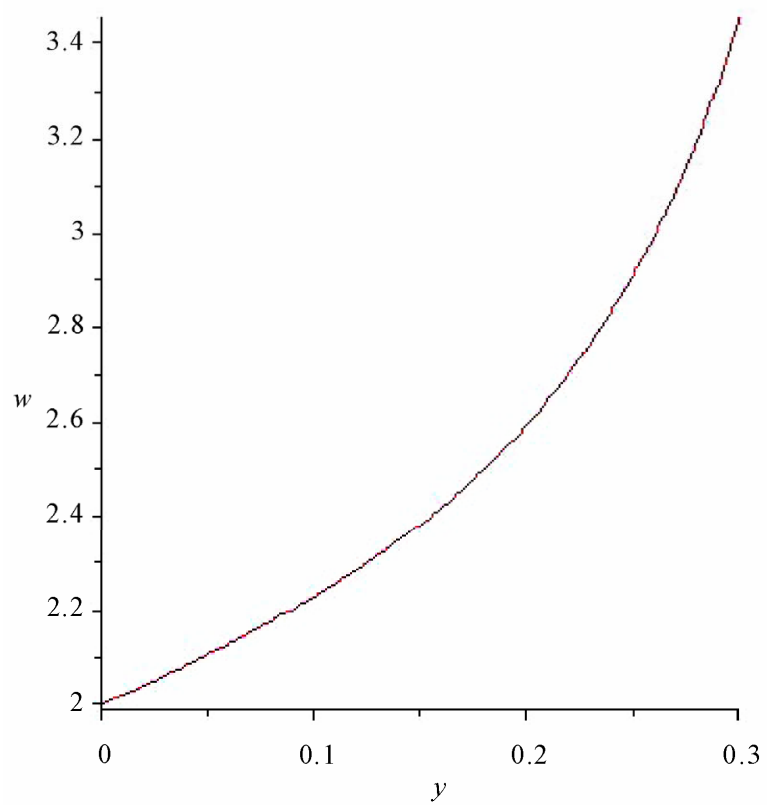

(a)

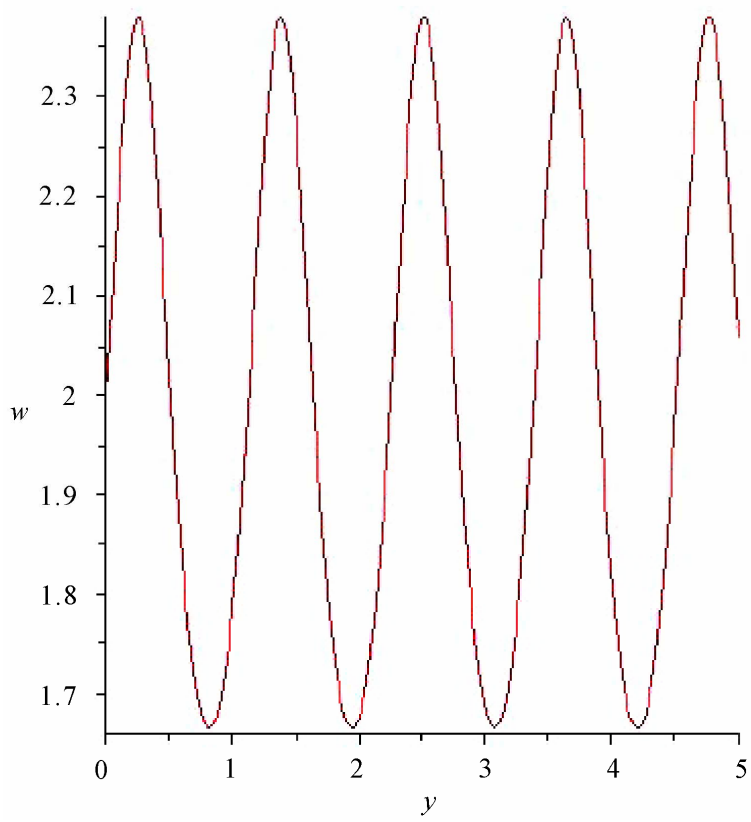

(b)

Figure 10. Numerical solution of (75). 


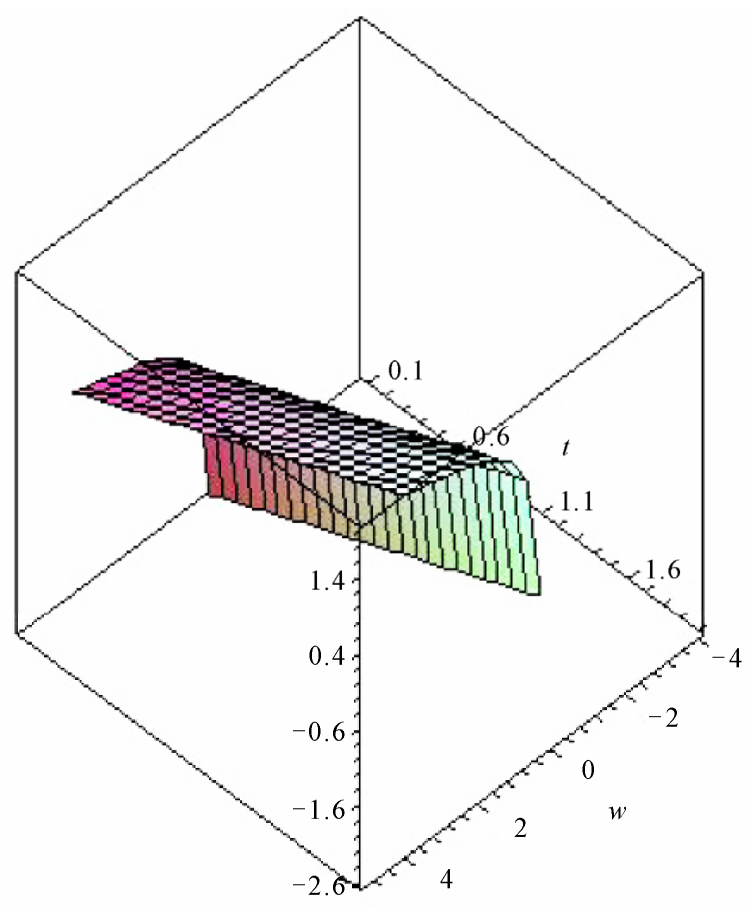

Figure 11. Soliton surface of (81).

this surface is illustrated in Figure 11.

\section{Conclusions}

We have discussed motion of curves in a plane and analysed nonlinear equations and related generalisations like vector using symmetry methods. These lead to exact solutions like travelling wave, soliton and other similarity solutions. Gaussian curvature equal zero and mean curvature don't equal zero lead to be surfaces cylinder of these equations.

\section{References}

[1] B. Kimia, A. Tannenbaum and S. Zucker, "On the Evolution of Curves via a Function of Curvature. I. The Classical Case," Journal Mathematical Analysis and Application, Vol. 163, No. 2, 1992, pp. 438-458. doi:10.1016/0022-247X(92)90260-K

[2] B. Kimia, A. Tannenbaum and S. Zucker, "Shapes, Shochs, and Deformations I: The Components of Two-Dimensional Shape and the Reaction-Diffusion Space," International Journal of Computer Vision, Vol. 15, No. 3, 1995, pp. 189-224. doi:10.1007/BF01451741

[3] C. Rogers and W. K. Schief, "Backlund and Darboux Transformations Geometry and Modern Application in Soliton Theory," Cambridge University press, Cambridge, 2002. doi:10.1017/CBO9780511606359

[4] M. do Carmo, "Differerntial Geometry of Curves and Surfaces," Prentice-Hall, Upper Saddle River, 1976.

[5] L. P. Eisenhart, "A Treatise on the Differential Geometry of Curves and Surfaces," Ginn, Boston, 1909.

[6] G. W. Bluman and S. Kumei, "Symmetries and Differential Equations," Springer, New York, 1989.

[7] P. J. Olver, "Applications of Lie Groups to Differential Equations," Springer, New York, 1986.

[8] P. Winternitz, "Lie Groups and Solutions of Nonlinear Partial Differential Equations, Integrable Systems, Quantum Groups, and Qunatum Field Theories," Kluwer Academic, Dordrecht, 1992.

[9] C. H. Gu, "Soliton Theory and Its Applications," Springer-Verlag, Berlin, 1995.

[10] C. H. Gu and H. S. Hu, "On the Determination of Nonlinear PDE Admitting Integrable System," Scientia Sinica, Series A, 1986, pp. 704-719. 